\title{
Ability of dyslexic and control teenagers to sustain attention and inhibit responses
}

\author{
Elisabeth Moores and Jackie Andrade \\ Department of Psychology, University of Sheffield, UK \\ DYSLEXIA AND SUSTAINED ATTENTION
}

Elisabeth Moores, Department of Neurological and Vision Sciences, Section of Human Physiology, University of Verona, Strada Le Grazie 8, Verona, Italy, I-37134

Email: mooresel@borgoroma.univr.it

Fax: +39045 580881

Tel: +390458098148

KEYWORDS: SART, ATTENTION, DYSLEXIA, INHIBITION, AUTOMATICITY, ATTENTION DEFICIT HYPERACTIVITY DISORDER, LEARNING DISABILITY, RESOURCES 


\begin{abstract}
Dyslexia and attentional difficulty have often been linked, but little is known of the nature of the supposed attentional disorder. The Sustained Attention to Response Task (SART: Robertson, Manly, Andrade, Baddeley and Yiend, 1997) was designed as a measure of sustained attention and requires the withholding of responses to rare (one in nine) targets. To investigate the nature of the attentional disorder in dyslexia, this paper reports two studies which examined the performance of teenagers with dyslexia and their age-matched controls on the SART, the squiggle SART (a modification of the SART using novel and unlabellable stimuli rather than digits) and the go-gap-stop test of response inhibition (GGST). Teenagers with dyslexia made significantly more errors than controls on the original SART, but not the squiggle SART. There were no group differences on the GGST. After controlling for speed of reaction time in a sequential multiple regression predicting SART false alarms, false alarms on the GGST accounted for up to 22\% extra variance in the control groups (although less on the squiggle SART) but negligible amounts of variance in the dyslexic groups. We interpret the results as reflecting a stimulus recognition automaticity deficit in dyslexia, rather than a sustained attention deficit. Furthermore, results suggest that response inhibition is an important component of performance on the standard SART when stimuli are recognised automatically.
\end{abstract}

\title{
INTRODUCTION
}

Developmental dyslexia is the most common developmental disorder, thought to affect around $4 \%$ of the human population (Badian, 1994; Jorm, Share, Maclean and Matthews, 1986). Defined by the World Federation of Neurology (1968) as "a disorder in children who despite conventional classroom experience, fail to attain the language skills of reading, writing and spelling commensurate with their intellectual abilities", dyslexia is usually diagnosed when there is a discrepancy of at least 18 months between reading and chronological age, with no immediately apparent cause in terms of deprivation, emotional difficulties or general low intelligence. Although a phonological deficit is well established as one of the key difficulties for children with dyslexia (see e.g. Snowling, 1997; Stanovich, 1988; Vellutino, 1979), and much is known about the nature of the deficit and its remediation, less is known about other aspects of dyslexia. For example, for many years there 
have been reports of concomitant attentional problems in children with dyslexia. Furthermore, it is a common clinical observation that dyslexia and attentional disorders frequently co-occur; around $15 \%$ of children with dyslexia are estimated to have concomitant attention deficit hyperactivity disorder (ADHD) and around 36\% of children with ADHD are estimated to have dyslexia (Shaywitz, Fletcher and Shaywitz, 1994). However, despite these links, and the impact that an attentional difficulty alone could have on a child's learning, little information exists on the exact nature of the reported attentional disorder. Moreover, there is little information on whether or not children with dyslexia but without ADHD show attentional difficulties. Previous studies have investigated various aspects of attention in different populations, including children with ADHD, dyslexia, and good and poor reading skills generally (e.g. Brannan and Williams, 1987; Dykman, Ackerman and Oglesby, 1979; Fischer and Weber, 1990; Hallahan, Kauffman and Ball, 1973; Milberg, Whitman and Galpin, 1981; Pearson and Lane, 1990; Pelham, 1979; Schacher, Logan, Wachsmuth and Chajcyzk, 1988; Sykes, Douglas and Morgenstern, 1973; Tarver, Hallahan, Kauffman and Ball, 1976). However, many results have been inconsistent and some experiments have been methodologically flawed. Some studies, for example, have used measures of attention which involve memory, an ability with which people with dyslexia are known to have difficulties.

The nature and extent of the purported attentional deficit in dyslexia therefore remains unclear. Three key aspects of attention include the abilities to focus, shift and sustain attention. Recent research (Moores, Nicolson and Fawcett, submitted) has suggested that these abilities may not be equally impaired in dyslexia; teenagers with dyslexia performed as well as controls on two focus attention conditions, but had significantly worse performance than controls on a shift attention condition. A rapid attention shifting deficit was hypothesised but not found, and thus it is difficult to make firm conclusions about whether or not the dissociation reflected pure attentional difficulties. On the basis of a second experiment, this dissociation was attributed to an automaticity deficit of the teenagers with dyslexia resulting in resource limitations. A crucial point is that none of the participants showed clinical evidence of ADHD. A 'split-half' analysis of the shift attention condition showed that groups with dyslexia could sustain their attention over that condition (about 15 minutes) as well as control groups, performance in both groups suffering similarly in the second half. However, recent neuroimaging evidence suggests that areas in the brain involved in sustained attention are active over periods as short as 40 seconds (Pardo, Fox and Raichle, 1991), rather than over tens of minutes as previously thought. It is therefore possible that 
local fluctuations and attentional lapses may characterise a deficit in sustained attention as validly as decreases in attention over long periods of time.

The Sustained Attention to Response Task (SART) was originally designed to counteract the difficulties of finding a true measure of sustained attention. Traditionally, continuous performance tests are used for this purpose, where participants have to monitor long sequences of stimuli and respond to infrequent targets. However, these tasks fail to tap shorter 'lapses of attention' and also often have problems with ceiling effects, leading researchers either to perceptually degrade targets or to load working memory in order to reduce high levels of performance. Fisk and Schneider (1981) make the distinction between automatic and controlled processing of stimuli (see also Schneider and Shiffrin, 1977). For tasks that have to be performed over relatively long periods, they show that performance decrements over time occur only when controlled processing is required and that, "Maximum vigilance decrements occur when subjects must continually and redundantly allocate control-processing resources." (Fisk and Schneider, p737). Robertson et al (1997) argue that controlled processing would be taxed more heavily if the automatic response set could be transferred to the non-targets, so that controlled processing was necessary to cancel out the automatic response. On the basis of this argument, the SART was developed, in which a response is required to every stimulus except for the targets, where responses must be withheld. The task runs over a period of under five minutes.

Robertson et al (1997) found that the SART was more sensitive to everyday attentional failures and 'lapses' of attention (as measured by self report questionnaires) than were continuous performance tests in both control and traumatic brain injured (TBI) patients. Performance on the SART was not predicted by performance on tests presumed to be sensitive to response inhibition: the Stroop, the Wisconsin Card Sorting Test and the Visual Elevator test. In addition, Robertson et al found that TBI patients were less likely than controls to slow down after an error, and that all participants were more likely to have 'lapsed' into an automatic way of responding (shown by faster reaction times) before an error. Performance of either group did not deteriorate significantly with time on task, such that the authors conclude that "local fluctuations in attention or 'lapses' may provide a better account of poor performance on this task than a simple decrement over time.” (p.755).

In an attempt to characterise better the attentional deficit associated with dyslexia, the first study assessed the performance of teenagers with dyslexia and matched (for age and IQ) 
controls on the SART. Given the reports that children with dyslexia have difficulty 'keeping on track' (Augur, 1985), we predicted that the teenagers with dyslexia in our study would be more prone to attentional lapses than controls and therefore perform less well on the SART.

\section{Experiment 1}

\section{$\underline{\text { Method }}$}

\section{Participants}

Fourteen participants with dyslexia had been diagnosed by a full psychometric assessment. They were of normal or above normal IQ [operationalised as IQ of 90 or more on the full scale WISC-III (Wechsler, 1976)] and without known primary emotional, behavioural or socio-economic problems. Each participant's reading age or spelling age (WORD tests) was at least 18 months behind their chronological age at time of initial diagnosis. Two age groups were used with mean ages 14.6 and 19.0 years (D15 and D19).

Fourteen normally achieving control participants had also been given a short-form psychometric assessment and obtained normal or above normal IQ and reading and spelling ages in line with or above their chronological age at time of assessment. Two age groups were used, approximately matched for chronological age with the group with dyslexia (C15 and $\mathrm{C} 19)$.

Participants had also all been assessed for clinical evidence of ADHD on the DSM IIIR (American Psychiatric Association, 1987). A score of at least 8 out of 14 markers of the disorder is required for clinical diagnosis. None of the participants showed evidence for ADHD. There were no significant differences between the score of the dyslexic and control groups $\left(\mathrm{F}_{1,26}=2.10\right.$, n.s.). All participants were given $£ 5$ for their co-operation in this study and for an unrelated study which followed this one. Psychometric data (means and ranges) for the four groups of participants are shown in Table 1.

[TABLE 1 ABOUT HERE]

\section{SART}

The SART program was written in PsyScope ${ }^{\mathrm{TM}}$ (Cohen, MacWhinney, Flatt \& Provost, 1993) by Robertson et al (1997). It visually presents a total of 225 digits ( 25 of each of the 
nine digits) on the computer screen over a 4.3 minute period. Each digit is presented for 250 milliseconds followed by a 900 millisecond mask. Participants use their preferred hand to press the mouse button in response to every digit except the 'target' digit 3, for which they are instructed to withhold responses. The target digit is distributed throughout the trials in a pre-fixed pseudorandom order which is the same for all participants. To enhance the need for processing the numerical value of the number rather than looking for a set 'shape' the digits are presented in one of five randomly allocated font sizes (48, 72, 94, 100 and 120 point: symbol font): between 12 and $29 \mathrm{~mm}$. The mask is a circle with a cross in the middle (diameter $29 \mathrm{~mm}$ ). Digits and mask are presented centrally on the screen in white, against a black computer screen positioned in front of the participant. A practice is given before the main condition consisting of 18 presentations of digits, two of which are 3.

\section{Procedure}

The method used was a replication of that used by Robertson et al (1997) using their SART program and run on an Apple Macintosh Performa 5200 computer. Participants were instructed to press the computer's mouse button as quickly as possible when a number appeared on the screen, except when that number was a three. They were informed that in between each digit there would be a cross which they should ignore. They were also told that the digits would vary in size, but that they should ignore this. Participants were then reminded to press the mouse button for all numbers except three, to attempt to do the task quickly, but also to try not to make errors. A short 18 digit practice was given before the main condition.

\section{Results}

The mean number of false alarms (FAs: non-withheld responses to 3) and mean reaction times (RTs) for the four groups are presented in Table 2. For simplicity, we report only those results for which $\mathrm{p}<0.05$ (two-tailed). Further details are available on request from the first author.

\section{[TABLE 2 ABOUT HERE]}

\section{i) Error analyses}

Robertson et al suggested that FAs on the SART reflect attentional lapse. A two-factor ANOVA found a main effect of group on FAs made $\left(\mathrm{F}_{1,24}=5.48, \mathrm{p}<0.05\right)$, with the teenagers 
with dyslexia making more FAs (14.3 vs. 10.6). There were no age effects or group-by-age interactions. Performance on the non-targets was good (dyslexic groups: 3\% misses, control groups: $2 \%$ misses) and did not differ by group or age.

\section{ii) Reaction time analyses}

Faster RTs may reflect a lesser degree of controlled attention and therefore more FAs. A two-factor ANOVA showed no effect of group or age on correctly pressed RTs and no group-by-age interaction. Similarly, group and age did not have a significant effect on RT of FAs and there was no significant group-by-age interaction.

Robertson et al interpreted a larger variability in RT in TBI patients as additional evidence that the SART reflects ability to maintain consistent performance. In a two-factor ANOVA using the standard deviation of each individual's RTs as dependent variable, the effect of group was not significant, but there was a main effect of age $\left(\mathrm{F}_{1,24}=7.49, \mathrm{p}<0.05\right)$, with younger participants being more variable. There was no group-by-age interaction.

Robertson et al found that, in contrast to controls, TBI patients tended not to slow down following a FA. A three-factor ANOVA therefore investigated effects of age, group and time (with respect to a target digit: pre-/ post-FA) on the mean RT of the four responses either side of a $\mathrm{FA}^{1}$. The effect of time was highly significant $\left(\mathrm{F}_{1,24}=14.40, \mathrm{p}<0.001\right)$, with post-FA responses being slower than pre-FA. Time also interacted with group $\left(\mathrm{F}_{1,24}=4.31, \mathrm{p}<0.05\right)$. Further analyses showed that whereas groups with dyslexia slowed down significantly after a FA $\left(F_{1,12}=17.84, p<0.005\right)$ control groups did not. There were no main group or age effects, but a significant group-by-age interaction did emerge $\left(\mathrm{F}_{1,24}=4.86, \mathrm{p}<0.05\right)$. Further analyses showed a main effect of age $\left(\mathrm{F}_{1,12}=4.81, \mathrm{p}<0.05\right)$ for the control groups only, with the $\mathrm{C} 19$ group being faster.

Robertson et al suggested that FAs could be predicted on the basis of pre-target digit RTs (the four responses before the target digit: 3). A three-factor ANOVA supported this

\footnotetext{
${ }^{1}$ The mean RT was in the vast majority of cases the mean of four responses, although in certain cases either two targets were too close together or participants failed to make responses to non-target digits. In these cases the mean of fewer responses was used.
} 
suggestion, showing faster responses before false alarms than before correctly withheld responses $\left(\mathrm{F}_{1,24}=16.90, \mathrm{p}<0.0005\right)$, but there were no main group or age effects.

\section{iii) The relationship between speed and accuracy}

Robertson et al discuss, but tentatively dismiss (on the basis of regression analyses with other measures) the notion that the slowing of RT following errors may arise because participants adopt a more conservative response criterion. However, our participants showed a strong negative relationship overall between mean RT and the number of errors made $(\mathrm{r}=-0.61, \mathrm{p}<0.001)$; i.e. a speed-accuracy trade-off. Analyses containing each group separately showed that this correlation was slightly stronger for the groups with dyslexia than the control groups $(\mathrm{r}=-0.72, \mathrm{p}<0.005$ vs. $\mathrm{r}=-0.63, \mathrm{p}<0.05)$, although the correlation coefficients do not differ significantly from each other (using Fisher's r' statistic, $z=0.41$ ). A significant correlation could also be seen between the number of FAs and the mean RT of responses before a correctly withheld response $(\mathrm{r}=-0.53, \mathrm{p}<0.005)$ showing that the main correlation is not merely due to the speeding of responses during attentional lapses (as indicated by a FA). An increased number of attentional lapses may be expected to increase the mean RT.

The relationship between RT and FAs may have had some bearing on the increased number of FAs made by the teenagers with dyslexia (although the groups did not differ on RT alone). Mean RT (for all correct responses) was therefore used as a covariate in a two-factor ANOVA investigating the effects of age and group on FAs. As expected, and reflecting the strong relationship between RT and FAs made, the effect of the covariate (RT) was highly significant $\left(\mathrm{F}_{1,23}=21.68, \mathrm{p}<0.0001\right)$. However, rather than being able to explain the increased number of FAs made in the group with dyslexia in terms of RT, the group effect was actually increased $\left(\mathrm{F}_{1,23}=10.85, \mathrm{p}<0.005\right)$. There were no main age effects or group-by-age interactions.

\section{$\underline{\text { Discussion }}$}

The main result of Experiment 1 was that the teenagers with dyslexia made more errors on the SART than their matched controls, even when RT was taken into consideration. This finding on its own suggested that, as predicted, the teenagers with dyslexia have difficulty sustaining attention and are more prone to attentional lapses even during a task that lasts under five minutes. However, the qualitatively normal pattern of performance in the groups with dyslexia prevented a straightforward conclusion and suggested that, at least with 
dyslexic teenagers, performance on the SART may reflect more than the ability to sustain attention.

Except for their high number of failures to withhold responses, the teenagers with dyslexia in Experiment 1 behaved like Robertson et al's controls; slowing down after making errors and showing only moderate variation in reaction times. Compared with their matched controls they also responded equally quickly and with similar variation in reaction times. Both groups showed a speed-accuracy trade-off during SART performance, but covarying for the effects of RT on the number of FAs enhanced rather than reduced group differences. Therefore, even if teenagers with dyslexia take as long as controls to make their responses, they make more errors. It seemed possible that the teenagers with dyslexia were under greater time pressure than the controls, despite their similar reaction times. Indeed, their similar reaction times were somewhat surprising and may be the key to explaining their high error rate.

Nicolson and Fawcett (1994) found that children with dyslexia were slower than controls at choice RT tasks, though not simple RT tasks. If the SART is considered essentially an omission choice RT task, the teenagers with dyslexia would appear to be responding at a speed beyond their true ability so their error rate is what might plausibly be expected from controls if they were encouraged to respond more quickly. If the SART is considered a simple RT task, one might still expect the teenagers with dyslexia to be slower because naming speed deficits are well established in both children and adults with dyslexia (e.g. Denckla and Rudel, 1976). If the teenagers with dyslexia took longer to convert the Arabic numerals e.g. ' 3 ' into the phonological ones, 'three' (see also Ellis, 1981), yet responded as quickly as the controls, they must have devoted less time to deciding whether to press the mouse button or whether to inhibit their response.

It is also plausible that an additional difficulty with response inhibition may contribute to the high error rates in the group with dyslexia, particularly given the links between dyslexia and ADHD and the suggestion that groups with ADHD show greater impulsivity and inability to withhold responses (e.g. Barkley, 1994). However, it should be emphasised that none of the teenagers in this study showed evidence of ADHD on the DSMIIIR scale. Robertson et al found no evidence that the SART reflected ability to inhibit responses in their patients, but they do admit that they were unable to demonstrate that it sits better with tests of sustained attention than with a 'pure' measure of response inhibition. 
Experiment 2 therefore explored two of the potential explanations of the dyslexic group's worse performance on the SART. To investigate the role of labelling in SART performance we used two SART versions: the standard version and one using non-labellable 'squiggles' in place of the digits. A test of response inhibition (the go-gap-stop test) was also introduced in order to (i) compare inhibition abilities of groups and (ii) investigate whether response inhibition is related to SART performance.

\section{Experiment 2}

\section{Method}

\section{Participants}

Participants fulfilled the same criteria as Experiment 1. Eighteen dyslexic and sixteen control teenagers took part in Experiment 2. Their psychometric details are shown in Table 3. Most of the participants who took part in Experiment 2 had previously taken part in Experiment 1 around 8 months earlier. This was not thought to have unduly affected the results; Manly et al (1999) have reported on the reliability of the results of the SART over time. Participants were given $£ 5$ for their co-operation in this experiment.

\section{[TABLE 3 ABOUT HERE]}

\section{SART AND SQUIGGLE SART}

Robertson et al's SART program was used as in Experiment 1.

Robertson et al's SART program was adapted to provide a 'squiggle SART' program by replacing the digits with squiggles created in a drawing program. The squiggles are shown in Figure 1. The target squiggle (shown within border) was chosen to be not easily labellable and other non-labellable squiggles were chosen as distractors to ensure the target could not be identified simply as 'the squiggle'. The presentation rate, practice session and duration of stimuli remained the same, as did the mask between the squiggles. In contrast to the original SART, squiggles did not vary in size over the trials and the order was completely random for each person. 


\section{THE GO-GAP-STOP TEST}

The go-gap-stop test (GGST) of response inhibition was designed by the first author and implemented in PsyScope (Cohen et al, 1993). Two possible stimuli could appear on the computer screen: a green circle and a red square. The green circle was intended as the signal for participants to 'go' and the red square as a signal to 'stop'. The green circle appeared on every trial for $125 \mathrm{~ms}$ after a random pause $(1500 \mathrm{~ms}, 2000 \mathrm{~ms}, 2500 \mathrm{~ms}$ or $3000 \mathrm{~ms}$ ) subsequent to the participant pressing the space bar and then keeping their hand on a velcro sticker positioned centrally on the desk in front of them. As soon as the green circle appeared (but not before) they were asked to hit the mouse button fixed $50 \mathrm{~cm}$ away from the velcro sticker either to the left or to the right of them (depending on whether they were left- or right-handed). However, if a red square subsequently appeared on the screen (again for $125 \mathrm{~ms}$ ), participants were asked to prevent themselves from pressing the mouse. Following the green circle, $1000 \mathrm{~ms}$ were allowed for a response before the trial finished, regardless of the trial type. The red square appeared on exactly half the trials. The crucial point was that the interstimulus interval between the green circle and the red square varied, so that sometimes it was easy to stop, but other times it was impossible. Whether or not the red square appeared (and at what point it appeared if it did) was random on any particular trial, although all participants received all of the possibilities at some point throughout the experiment. The fact that the red square never appeared on half the trials ensured participants followed the instruction to respond the green circle. The 35 possible interstimulus intervals $(0 \mathrm{~ms}, 13 \mathrm{~ms}$, $25 \mathrm{~ms}, 38 \mathrm{~ms}, 50 \mathrm{~ms} . . . . .450 \mathrm{~ms})^{2}$ between the green circle and the red square ensured that a strategy of waiting to check for the red square before responding to the green circle was unlikely to be successful, because it was never clear how long to wait. Moreover, the mean of the 35 reaction times to the green circle only (no-stop trials) gave an indication of any delays which may have occurred due to such strategies and such effects of reaction time were controlled for in the analysis. Participants were given a short practice at the task (5 trials) to ensure that they understood the instructions and procedure.

\section{Design and Procedure}

\footnotetext{
${ }^{2}$ A programming error added $500 \mathrm{~ms}$ to three delays, thus $12.5 \mathrm{~ms}$ became $512.5 \mathrm{~ms}, 37 \mathrm{~ms}$ became $537 \mathrm{~ms}$ and $62 \mathrm{~ms}$ became $562 \mathrm{~ms}$.
} 
The order in which the two SART programs were administered was counterbalanced over age and group to avoid any order effects. The GGST was always administered last. The whole procedure lasted under 30 minutes. For some participants the squiggle SART practice had to be repeated because they had difficulty remembering which squiggle was the target. It was ensured that all participants were sure of the target before commencing the main test. Observation of participants whilst completing the test suggested that even those who made a lot of mistakes were nevertheless well aware of when they had. For the SART programs, participants instructions were the same as in Experiment 1, exchanging a picture of the appropriate squiggle for ' 3 ' where appropriate. Instructions for the GGST are detailed in the description of the program.

\section{$\underline{\text { Results }}$}

The mean number of false alarms (FAs: non-withheld responses to 3 or the target squiggle) and mean reaction times (RTs) for the four groups in either SART program are presented in Table 4. For both the standard and the squiggle SART the same analyses were performed as for Experiment 1. Therefore, for brevity the tests used and the reasoning behind each analysis will not be reported again (see Experiment 1 results for this information). Both SARTs and the GGST were then considered together. An overview of the main results is provided in the discussion.

\section{[TABLE 4 ABOUT HERE]}

\section{Standard SART}

In summary, the main results from Experiment 1 were replicated in the standard SART except that (i) dyslexic groups now showed larger variability than the controls in their RTS (ii) both groups now slowed down equally after FAs.

\section{i) Error analyses}

The dyslexic groups made more FAs than the control groups $\left(\mathrm{F}_{1,30}=4.73, \mathrm{p}<0.05\right)$. Age and group-by-age effects on FAs were not significant. Misses to non targets were rare 
(dyslexic groups 2\%, control groups 1\%) and generated no significant group, age or group-by-age interaction effects.

\section{ii) Reaction time analyses}

For correct RTs there were no significant group, age, or group-by-age effects Similarly, false alarm RTs generated no significant effects.

In contrast to results from Experiment 1, within-subject variability of RTs generated a main effect of group $\left(\mathrm{F}_{1,30}=5.34, \mathrm{p}<0.05\right)$, with dyslexic groups being more variable. There was also a main effect of age $\left(\mathrm{F}_{1,30}=5.20, \mathrm{p}<0.05\right)$, with younger participants being more variable. There was no group-by-age interaction.

The response style analysis revealed faster RTs before FAs than after FAs $\left(F_{1,30}=9.66\right.$, $\mathrm{p}<0.005)$. There was also a significant interaction of time with age $\left(\mathrm{F}_{1,30}=6.07, \mathrm{p}<0.05\right)$, with younger groups slowing more after a FA, and a main age effect $\left(\mathrm{F}_{1,30}=5.61, \mathrm{p}<0.05\right)$ with older groups having faster RTs overall. There were no group or group-by-age interaction effects.

The error prediction analysis confirmed that RTs were slower before a correctly withheld response than an FA response $\left(\mathrm{F}_{1,30}=22.89, \mathrm{p}<0.0001\right)$. No other effects were significant.

\section{iii) The relationship between speed and accuracy}

There was a significant negative correlation between mean RT and FAs made ( $r=-0.49$, $\mathrm{p}<0.005)$. This correlation was slightly stronger for the groups with dyslexia than the control groups $(\mathrm{r}=-0.61, \mathrm{p}<0.05$ vs. $\mathrm{r}=-0.56, \mathrm{p}<0.05)$ and overall a significant correlation remained even when only the mean RT of responses before a correctly withheld response were considered $(\mathrm{r}=-0.39, \mathrm{p}<0.05)$.

RT proved to be a significant covariate $\left(\mathrm{F}_{1,29}=14.10, \mathrm{p}<0.001\right)$ when added to the original ANOVA comparing number of FAs made. The effect of group was enhanced $\left(\mathrm{F}_{1,29}=9.91, \mathrm{p}<0.005\right)$ by use of the covariate. Effects of age and the interaction effect between group and age were not significant. 


\section{Squiggle SART}

Squiggle SART results varied from those of the standard SART presented in Experiment 1 because (i) no group difference in FAs was observed in the main analysis, (ii) age had no effect on RT variability, (iii) participants were significantly faster after rather than before FAs, (iv) this latter effect no longer interacted with group, and (v) ANCOVA analysis on FAs enhanced the group effect to significance.

\section{i) Errors made}

Group, age and group-by-age effects were not significant for the number of FAs made. Similarly, investigation of number of misses to non-targets yielded no significant effects on these measures (dyslexic groups 4\%, control groups 3\%).

\section{ii) Reaction time analyses}

There were no significant group, age, or group-by-age interaction effects for either correct RTs, false alarm RTs or within-subject variability in RTs.

For the response style analysis there was a main effect of time $\left(\mathrm{F}_{1,30}=4.38, \mathrm{p}<0.05\right)$ but, in contrast to previous analyses, RTs were faster post- than pre-FAs. There were no group, age or group-by-age interaction effects.

The error prediction analysis showed faster RTs before FAs than before CWs $\left(\mathrm{F}_{1,30}=25.03, \mathrm{p}<0.0001\right)$. No group, age, or group-by-age interactions were found.

\section{iii) The relationship between speed and accuracy}

There was an overall negative correlation between mean RT and FAs made $(r=-0.65$, $\mathrm{p}<0.0001$ ). This was slightly stronger (n.s.) for the groups with dyslexia than the control groups $(\mathrm{r}=-0.84, \mathrm{p}<0.0001$ vs. $\mathrm{r}=-0.55, \mathrm{p}<0.05)$ and somewhat diminished, though still significant, even when only the mean RT of responses before a correctly withheld response were considered $(r=-0.34, p<0.05)$.

In an ANCOVA analysis the effect of RT as covariate had a highly significant effect $\left(\mathrm{F}_{1,29}=27.44, \mathrm{p}<0.001\right)$ on the number of FAs made and the effect of group was also now significant $\left(\mathrm{F}_{1,29}=7.36, \mathrm{p}<0.01\right)$, with the dyslexic groups making more errors. Effects of age, and the interaction effect between group and age, were not significant. 


\section{SART vs. Squiggle SART}

Because we originally predicted that labelling aspects of the standard SART were important in determining performance, and that control groups' superior labelling abilities may have contributed to their superior performance, independent two-factor ANOVAs investigating effects of age and SART version on errors and RTs were carried out for dyslexic and control groups independently. For the control group, SART version had a highly significant effect on errors made, with considerably more errors made on the squiggle SART $\left(\mathrm{F}_{1,16}=25.25, \mathrm{p}<0.0001\right)$. The same was true for RTs, with squiggle RTs being significantly slower $\left(\mathrm{F}_{1,16}=15.47, \mathrm{p}<0.005\right)$. However, for the dyslexic groups, SART version had no significant effect on the number of errors made, although their mean RTs were slower with the squiggle $\operatorname{SART}\left(\mathrm{F}_{1,14}=11.38, \mathrm{p}<0.005\right)$.

In a three-factor ANOVA (investigating factors of group, age and SART version on the number of FAs made) the main group effect narrowly failed to reach significance $\left(\mathrm{F}_{1,30}=3.77\right.$, $\mathrm{p}=0.06)$ and there was a significant main effect of SART version $\left(\mathrm{F}_{1,30}=14.24, \mathrm{p}<0.001\right)$, with the squiggle SART and the dyslexic groups producing more errors. The interaction between SART version and group was not significant. No other effects were significant.

\section{Go-Gap-Stop Test}

The mean numbers of FAs and mean RTs on "go" trials for each group are shown in Table 5.

\section{[TABLE 5 ABOUT HERE]}

The number of FA responses (trials on which participants could not stop, but should have done) were counted for each participant. These were analysed using an ANCOVA, investigating effects of age and group after covarying for mean RT when no stop-signal was presented. As would be expected, the effect of mean RT was highly significant $\left(F_{1,26}=16.15\right.$, $\mathrm{p}<0.0005)$. There were no group, age, or group-by-age effects. Teenagers in the control group failed to stop on as many occasions (mean $=16.2$ ) as those in the dyslexic group (mean = 15.1). 


\section{Relationship between the SARTs and the GGST}

To elucidate any relationship between performance on the SART and response inhibition ability, correlations between FAs on the SART and GGST were performed for each SART task and for each group independently. For the standard SART, there was no correlation for the dyslexic group ( $r=-0.03$, n.s.), but a significant positive correlation for the controls $(\mathrm{r}=0.61, \mathrm{p}<0.01)$. Similarly, for the squiggle SART there was a significant positive correlation for the control $(\mathrm{r}=0.49, \mathrm{p}<0.05)$, but not the dyslexic group $(\mathrm{r}=-0.01, \mathrm{n} . \mathrm{s})$.

However, since speed-accuracy trade-offs found on both tasks are able to explain a relationship between the two tasks without consideration of response inhibition (some people might always respond quickly and so make more mistakes on both tasks) sequential multiple regressions were performed for each group and for each SART version independently. These analyses statistically controlled first for mean RT on the SART task and then investigated how much extra variance FAs on the GGST could account for. For the controls, on the standard SART, 39\% of the variance in FAs (adj:35\%) ${ }^{3}$ was accounted for by mean RT on the SART and $61 \%(\operatorname{adj}: 56 \%$ ) by mean RT on the SART and GGST FAs together: thus an increase of over $20 \%$ from the addition of performance measures from a response inhibition test. On the squiggle SART for the controls, 30\% (adj:26\%) of the variance was accounted for by mean RT on the squiggle SART and $41 \%$ (adj: 32\%) by this and GGST FAs together: thus a smaller increase of between 6 and 11\%. For the dyslexic groups, 44\% (adj:40\%) of the variance was accounted for by mean RT on the standard SART task, but nothing added by GGST FAs (R squared=44\%, adj: 36\%). On the squiggle task, dyslexic mean RT explained $70 \%$ (adj:68\%) of the variance in FAs and response inhibition added at most a negligible amount (71\% - adj 67\% - for both measures together).

\section{General discussion}

The main findings of the two experiments are that: (i) teenagers with dyslexia made more FAs on the SART but their response rates were as fast as those of the control group and qualitatively normal (though somewhat more variable in Experiment 2), (ii) the group

${ }^{3}$ Both Multiple R square and adjusted R square will be reported for the multiple regressions, because of the small sample sizes used (in a small sample with several independent variables, $\mathrm{R}$ square can be large due to chance fluctuations in the data: adjusted $\mathrm{R}$ square gives an estimate of the proportion of variance which might be expected to be explained should the study be repeated). 
difference in FAs was not significant when the SART used squiggles rather than digits as stimuli, although covarying for RT did produce a group difference, (iii) the GGST showed no group differences in response inhibition, and (iv) contrary to Robertson et al's (1997) findings with other tests thought to reflect response inhibition, there was a strong relationship between GGST and SART performance for the control, but not the dyslexic, groups.

At first glance, the finding that the teenagers with dyslexia were impaired on the standard SART but not the squiggle SART suggests that were poorer at recognising the digits in the standard SART, but did not have a deficit in sustained attention. However, the different relationships observed between SART performance and response inhibition suggest that the SART is a more complex task than originally indicated by Robertson et al's (1997) data. We will therefore begin by discussing what the control group's performance reveals about the SART.

For the control group, response inhibition failures (FAs on the GGST) correlated strongly with SART FAs and explained a considerable proportion of the variance in SART FAs even after SART response times had been partialled out. This shows that the ability to prevent a planned motor response being completed is important for avoiding errors on the SART. Note that Robertson et al (1997) argued that the SART measures the sustained attention needed to overcome automatic responding to digits. Our data show that the ability to inhibit responses that have already been initiated is also important. However, we propose that response inhibition is only important when the stimuli are recognised automatically. For control participants, digits should be well and immediately recognised, allowing rapid responding that might soon be expected to become completely automatised. Automatic responding requires little or no attention and is fast, therefore response inhibition might be necessary to withhold an automated response that has escaped detection by attentional processes. In the squiggle SART, the digits were replaced by novel squiggles which required learning and therefore controlled processing, at least in the early stages of the task. If response inhibition is only important to override automatic responses, then it may only have become important to performance in the later stages of the squiggle SART. Non-automatic recognition of squiggles in the early stages of the squiggle SART can thus account for the weaker, though still statistically significant, association between the squiggle SART and the GGST. The poorer performance of controls on the squiggle SART compared to the standard SART can also be attributed to non-automatic recognition of squiggles making the whole task more resource consuming. 
For the control group, response inhibition contributed less to performance on the squiggle SART than the standard SART. If response inhibition is only needed for withholding automatic responses, and if automatic responding is critical for measuring sustained attention, then the squiggle SART may be a less sensitive measure of sustained attention than the standard SART. We suggest that the decreased relationship between response inhibition and SART FAs in the squiggle condition reflects low levels of automaticity, particularly in the early stages of the task. Allowing participants a longer practice session on the squiggle SART may be a way around this problem in future. The larger number of FAs on the squiggle SART may reflect a different response criterion, rather than greater difficulty sustaining attention. Both tasks showed a speed-accuracy trade-off, suggesting that at least some errors were made because participants accepted a relatively high error rate in order to continue responding quickly. Thus, in conjunction with Robertson et al's data, the data from our control group suggest that sustained attention, response inhibition and speed-accuracy trade-off all contribute to the number of errors made on the SART. However, whether sustained attention and response inhibition contribute independently to SART performance remains a question for further research.

We now move onto explaining the pattern of performance shown by the teenagers with dyslexia. In both experiments, the dyslexic group made more errors on the standard SART than did the controls, but they were not impaired on the squiggle SART. As already discussed in the discussion section of Experiment 1, one explanation is that phonological processing problems (see Ellis, 1981), rather than a sustained attention deficit, caused their poorer performance on the standard SART. This raises the question of why the dyslexic teenagers did not slow down to compensate for the longer time they needed to process the digits. They showed a similar speed-accuracy trade off to the controls, suggesting they were equally able to monitor and control their performance. They also performed as well as the controls on the GGST, showing no difficulty in response inhibition. One possibility is higher motivation than the control group. Therefore slower phonological processing, combined with fast responding due to higher motivation, remains one explanation of the dyslexic group's poorer performance on the standard SART.

However, interestingly, the GGST data for the dyslexic group showed no contribution at all of response inhibition ability to SART performance. We argued above that response inhibition is only needed to prevent completion of automatised responses. We now extend this argument to suggest that the dyslexic group may have never reached the point of 
responding automatically on either SART task. For the standard SART, this may be attributed to their phonological processing difficulty but this explanation cannot apply to the squiggle SART because the stimuli were not nameable. Therefore the data from both tasks may be better explained by a more general automaticity deficit in dyslexia, as proposed by Nicolson and Fawcett (1990). A stimulus recognition automaticity deficit can explain why the squiggle SART is no more difficult for the dyslexic groups than the standard SART; recognition of neither digits nor squiggles has been automatised, so neither is more difficult than the other and response inhibition is unimportant in both. Whereas the control participants may have begun responding automatically as they became more familiar with the stimuli on the squiggle SART, the teenagers with dyslexia remained in a controlled processing mode for the duration of the task. The digit/ squiggle manipulation has therefore not only altered the phonological component of the task, but also a component of previously learned symbol recognition. This is almost inevitable because learned symbols tend to be nameable.

We initially set out to discover whether the teenagers with dyslexia have difficulty sustaining attention. The lack of a group difference in false alarms on the squiggle SART suggests that they do not. However, this conclusion must be treated with caution for two reasons. First, the squiggle SART may not measure sustained attention unless responding becomes automated, and the lack of importance of response inhibition suggests it does not for the dyslexic group. Second, when the squiggle SART FAs were reanalysed with reaction time as a covariate, a group difference did emerge. However, assuming that there is no just cause for covarying for reaction time ${ }^{4}$, then our data show that the dyslexic group performed as well on the SART as the control group, providing the task required no phonological processing or learned symbol recognition. However, we attribute their equivalent performance to the poor

4 Whether or not RT should be covaried for depends on whether you believe (i) that periods of attentional lapse cause faster RT and errors, or (ii) that faster reaction times cause errors regardless of attentional state (i.e. a speed-accuracy trade-off). If it is believed that attentional lapses alone increase RT [option (i)], then covarying for RT removes the very effects of interest because RT and FAs are statistically and logically inseparable. However, if it is believed that going too fast on the SART causes errors then either (hard version) the SART is not a measure of attention at all, merely of one's criterion with respect to speed-accuracy, or (soft version) although the SART is a good measure of attentional lapse, a speed-accuracy trade-off is also involved. We argued above that the SART measures a combination of attention, response-inhibition and speed-accuracy trade off [see also Manly et al (1999)]. Since it is impossible to separate RTs that reflect speed-accuracy trade-off from those that reflect attentional lapse, covarying for RT presents a tricky problem. It reduces the noise due to different response criteria but also removes some of the variance of interest i.e. the effects of attentional lapse. The second main issue with respect to the use of covariates is whether it is fair to match groups on reaction time at all. Given the evidence already discussed for slower reaction times for dyslexic groups on some tasks, matching groups on the basis of reaction time may be completely unjustified. 
automatisation of the squiggle SART by both groups, rather than to their equal sustained attention abilities.

We conclude that the SART paradigm depends on automatic recognition of stimuli leading to automated responding and that, because it is likely that neither digits nor squiggles are recognised automatically in dyslexic teenagers, the SART paradigm is not a good one for dyslexia research into attentional lapses. Furthermore, when stimuli are recognised automatically, response inhibition is an important component of the SART, although sustained attention is likely to be necessary in order to inhibit responses. When stimuli are novel and not recognised automatically, there is a speed-accuracy trade-off but response inhibition is less important. Results of the Go-Gap-Stop Test suggest no response inhibition deficit in dyslexia. 


\section{ACKNOWLEDGEMENTS}

We are grateful to Tom Manly of the MRC Cognition and Brain Sciences Unit in Cambridge for sending us a copy of the standard SART program. We also thank anonymous reviewers and Rod Nicolson for helpful comments on the paper and to all the participants for their co-operation. 


\section{REFERENCES}

American Psychiatric Association (1987). Diagnostic and Statistical Manual of Mental Disorders, 3rd edition, Revised. Washington DC: American Psychiatric Association.

Augur, J. (1985). Guidelines for teachers, parents and learners. In M.J. Snowling (Ed.), Children's written language difficulties: Assessment and management. London: Routledge. p147-169.

Badian, N.A. (1994). Preschool prediction: Orthographic and phonological skills and reading. Annals of Dyslexia, 44, 3-25.

Barkley, R.A. (1994). Delayed responding and attention deficit hyperactivity disorder: toward a unified theory. In D.K. Routh (Ed.), Disruptive behavior disorders in children: Essays in honor of Herbert Quay (p11-57). New York: Plenum. Cited in Chapter 4 in Attention Memory and Executive Function, G.R.Lyon and N.A.Krasnegor (eds.), Paul Brookes Publishing Co., Maple Press Company, York: Pennsylvania.

Brannan, J.R. and Williams, M.C. (1987). Allocation of visual attention in good and poor readers. Perception and Psychophysics, 41, 23-28.

Cohen, J., MacWhinney, B., Flatt, M. and Provost, J. PsyScope: An interactive graphic system for designing and controlling experiments in the psychology laboratory using Macintosh computers. Behavior Research Methods, Instruments and Computers, 25, 257-271.

Denckla, M.B. and Rudel, R.G. (1976). Rapid 'Automatized' Naming (R.A.N.): Dyslexia differentiated from other learning disabilities. Neuropsychologia, 14, 471-479.

Dykman, R.A., Ackerman, P.T. and Oglesby, D.M. (1979). Selective and Sustained Attention in hyperactive, learning-disabled and normal boys. Journal of Nervous and Mental Disease, 167, 288-297.

Ellis, N. (1981). Visual and name encoding in dyslexic children. Psychological Research, 43, 201-18. 
Fisk, A.D. and Schneider, W. (1981). Controlled and automatic processing during tasks requiring sustained attention: A new approach to vigilance. Human Factors, 23, 737-750.

Fischer, B. and Weber, H. (1990). Saccadic reaction times of dyslexic and age-matched normal subjects. Perception, 19, 805-818.

Hallahan, D.P., Kauffman, J.M. and Ball, D.W. (1973). Selective attention and cognitive tempo of low achieving and high achieving sixth grade males. Perceptual and Motor Skills, 36, 579-583.

Jorm, A.F., Share, D.L., Maclean, R. and Matthews, D. (1986). Cognitive factors at school entry predictive of specific reading retardation and general reading backwardness: A research note. Journal of Child Psychology and Psychiatry and Allied Disciplines, 27, $45-54$.

Manly, T., Robertson, I.H., Galloway, M. and Hawkins, K. (1999). The absent mind: further investigations of sustained attention to response. Neuropsychologia, 37, 661-670.

Milberg, V.W., Whitman, R.D. and Galpin, R. (1981). Selective attention and laterality in good and poor readers. Cortex, 17, 571-82.

Moores, E., Nicolson, R.I. and Fawcett, A.J. (submitted). Attentional deficits in dyslexia: Evidence for an automatisation deficit?

Nicolson, R.I. and Fawcett, A.J. (1990). Automaticity: A new framework for dyslexia research? Cognition, 35, 159-182.

Nicolson, R.I. and Fawcett, A.J. (1994). Reaction times and dyslexia. Quarterly Journal of Experimental Psychology, 47A, 29-48.

Pardo, J.V., Fox, P.T. and Raichle, M.E. (1991). Localization of a human system for sustained attention by positron emission tomography. Nature, 349, 61-64.

Pearson, D.A. and Lane, D.M. (1990). Reorientation in hyperactive and non-hyperactive children: Evidence for developmentally immature attention? In J.T.Enns (Ed.) The Development of Attention: Research and Theory. Elsevier Science: Holland. 
Pelham, W. (1979). Selective attention deficits in poor readers? Dichotic listening, speeded classification, and auditory and visual central and incidental learning tasks. Child Development, 50, 1050-61.

Rabbitt, P.M. (1979). How old and young subjects monitor and control responses for accuracy and speed. British Journal of Psychology, 70, 305-311.

Robertson, I.H., Manly, T., Andrade, J., Baddeley, B.T. and Yiend, J. (1997). 'Oops!': Performance correlates of everyday attentional failures in traumatic brain injured and normal subjects. Neuropsychologia, 35, 747-758.

Schacher, R., Logan, G., Wachsmuth, R. and Chajcyzk, D. (1988). Attaining and maintaining preparation: A comparison of attention in hyperactive, normal and disturbed control children. Journal of Abnormal Child Psychology, 16, 361-378.

Schneider, W. and Shiffrin, R.M. (1977). Controlled and automatic human information processing:1. Detection, search and attention. Psychological Review, 84, 1-66.

Shaywitz, S.E., Fletcher, J.M. and Shaywitz, B.A. (1994). Issues in the definition and classification of attention deficit disorder. Topics in Language Disorders, 14, 1-25.

Snowling, M. (1987). Dyslexia: A Cognitive Developmental Perspective. Blackwell, Oxford.

Stanovich, K.E. (1988). Explaining the differences between the dyslexic and the garden variety poor reader: The phonological-core variable-difference model. Journal of Learning Disabilities, 21, 590-612.

Sykes, D.H., Douglas, V.I., and Morgenstern, G. (1973). Sustained attention in hyperactive children. Journal of Child Psychology and Psychiatry, 14, 213-220.

Tarver, J.G., Hallahan, D.P., Kauffman, J.M. and Ball, D.W. (1976). Verbal rehearsal and selective attention in children with learning disabilities. Journal of Experimental Child Psychology, 22, 375-385.

Vellutino, F.R. (1979). Dyslexia: Theory and Research. Cambridge, MA: MIT Press.

Wechsler, D. (1976). Wechsler Intelligence Scale for Children - 3rd edition(WISC III). Sidcup, UK; The Psychological Corporation, Europe. 
World Federation of Neurology (1968). Report of research group on dyslexia and world illiteracy. Dallas: WFN. 
Table 1. Psychometric data for each group of participants in Experiment 1. ( $D=$ dyslexic, $C=$ control). Range shown in parentheses.

\begin{tabular}{|c|c|c|c|c|c|}
\hline Group & n & Mean Age & Mean IQ & Mean RA & Mean ADHD \\
\hline D15 & 8 & $14.6(13.5-15.2)$ & $113(96-134)$ & $13.4(9.3-17)^{*}$ & $1.0(0-6)$ \\
\hline C15 & 9 & $15.0(13.8-16.0)$ & $116(101-129)$ & $16.2(14.0-17+)$ & $0.1(0-1)$ \\
\hline D19 & 6 & $19.0(17.3-20.9)$ & $115(101-131)$ & $12.8(9.3-16.0)$ & $0.3(0-1)$ \\
\hline C19 & 5 & $18.8(17.8-19.5)$ & $114(96-130)$ & $17+$ & 0.0 \\
\hline
\end{tabular}

* Three of the participants in this group had caught up with their reading since time of diagnosis. These were teenagers of high IQ. Their spelling ages remained significantly lowered. 
Table 2. Mean False Alarm (FA: non-withheld responses to $3 \mathrm{~s}$ ) and Reaction Time (RT: in milliseconds) data for the four groups (standard deviations shown in parentheses) in Experiment 1.

\begin{tabular}{|l|l|l|l|}
\cline { 2 - 4 } \multicolumn{1}{c|}{} & FAs (max. 25) & RTs to non-targets & RTs to targets (FAs) \\
\hline D15 & $14.8(2.8)$ & $326(50)$ & $278(45)$ \\
\hline C15 & $10.3(4.6)$ & $373(96)$ & $282(37)$ \\
\hline D19 & $13.7(3.3)$ & $334(54)$ & $295(43)$ \\
\hline C19 & $11.0(4.7)$ & $297(49)$ & $253(40)$ \\
\hline
\end{tabular}


Table 3. Psychometric data for each group of participants in Experiment 2. $(D=d y s l e x i c$, $C=$ control). Range shown in parentheses.

\begin{tabular}{|c|c|c|c|c|c|}
\hline Group & $\mathbf{n}$ & Mean Age & Mean IQ & Mean RA & Mean ADHD \\
\hline D15 & 9 & $15.5(14.2-16.4)$ & $107(90-126)$ & $13.2\left(9.0-17^{\S}\right)$ & $1.0(0-6)$ \\
\hline C15 & 7 & $15.8(14.5-16.9)$ & $116(101-129)$ & $16.4(13.3-17)$ & $1.0(0-5)$ \\
\hline D19 & 9 & $19.6(18.0-21.6)$ & $119(101-131)$ & $14.5(9.6-17)$ & $1.0(0-5)$ \\
\hline C19 & 9 & $19.7(18.5-20.2)$ & $118(96-129)$ & $17+$ & 0.0 \\
\hline
\end{tabular}

$\S_{\text {The anomalous score of } 17 \text { derives from one dyslexic participant who has been }}$ diagnosed some years previously and had subsequently made exceptional progress in reading. Nonetheless, his spelling was very poor. In addition one of the controls had fallen behind in his reading somewhat since leaving education. Perhaps of interest is that this person was also the control with the highest ADHD score. This particular participant has been in the control panel at the University of Sheffield for several years, so was left in the analyses as a control. 
Table 4. Mean FAs and mean correct RTs made in the standard and squiggle SART versions in Experiment 2 (standard deviations shown in parentheses).

\begin{tabular}{|l|l|l|l|l|}
\hline & FAs: standard & FAs:squiggle & RTs:standard & RTs:squiggle \\
\hline D15 & $14.2(6.26)$ & $14.4(6.33)$ & $320(64)$ & $364(97)$ \\
\hline C15 & $8.9(2.76)$ & $11.3(2.55)$ & $330(56)$ & $374(65)$ \\
\hline D19 & $13.6(3.78)$ & $15.9(3.44)$ & $313(85)$ & $359(81)$ \\
\hline C19 & $11.2(6.38)$ & $14.11(5.28)$ & $265(52)$ & $311(62)$ \\
\hline
\end{tabular}


Table 5. Table showing the mean number of FAs and the mean RT on no stop trials for the four groups of participants in Experiment 2(standard deviations shown in parentheses).

\begin{tabular}{|l|l|l|}
\hline & Mean FAs & Go Mean RT \\
\hline D15 & $15.6(7.44)$ & $687(92)$ \\
\hline C15 & $14.2(8.26)$ & $682(37)$ \\
\hline D19 & $14.6(4.43)$ & $682(80)$ \\
\hline C19 & $18.1(7.91)$ & $633(142)$ \\
\hline
\end{tabular}


Figure 1. The Squiggles (target enclosed in box)
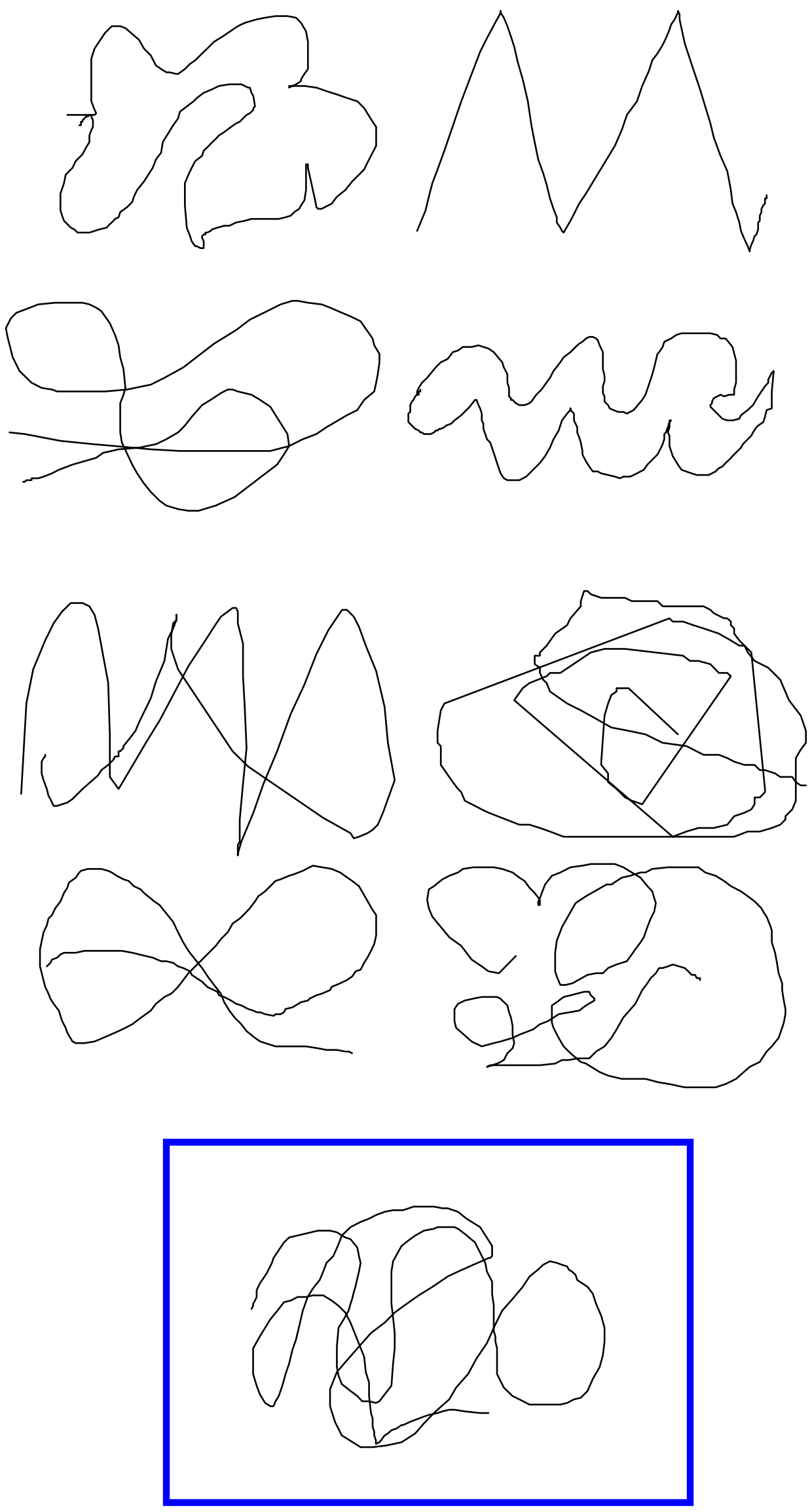